Research Paper

\title{
Diagnostic accuracy of inflammatory markers for distinguishing malignant and benign ovarian masses
}

\author{
Wan Kyu Eo ${ }^{\#}$, Ki Hyung Kim ${ }^{2 \bowtie *}$, Eun Joo Park ${ }^{3 凶 *}$, Heung Yeol Kim ${ }^{\#}$, Hong-bae Kim5 ${ }^{5}$ Suk Bong Koh6, \\ Jeong Namkung ${ }^{7}$ \\ 1. Department of Internal Medicine, College of Medicine, Kyung Hee University, Seoul, Korea \\ 2. Department of Obstetrics and Gynecology, Pusan National University School of Medicine; Biomedical Research Institute, Pusan National University \\ Hospital, Busan, Korea. \\ 3. Department of Obstetrics and Gynecology, Eulji Medical Center, Eulji University. Seoul, Korea \\ 4. Department of Obstetrics and Gynecology, College of Medicine, Kosin University, Busan, Korea. \\ 5. Department of Obstetrics and Gynecology, Kangnam Sacred Heart Hospital, Hallym University Medical Center, Hallym University College of Medicine, \\ Seoul, Korea. \\ 6. Department of Obstetrics and Gynecology, Catholic University of Daegu, School of Medicine, Daegu, Korea. \\ 7. Department of Obstetrics and Gynecology, Catholic University, Seoul, Republic of Korea. \\ \# Eo WK and Kim HY equally contributed to this article for first author. \\ * Both Kim KH and Park EJ equally contributed to this article for correspondence
}

$\square$ Corresponding author: Ki Hyung Kim, M.D., Ph.D., Department of Obstetrics and Gynecology, Pusan National University School of Medicine; Biomedical Research Institute, Pusan National University Hospital, Busan 602-739, Korea; Phone: +82-51-990-6226; Fax: +82-51-990-3300; e-mail: kihyungkimpnu@gmail.com and Eun Joo Park, M.D., Ph.D., Department of Obstetrics and Gynecology, Eulji Medical center, Eulji University , 34, Hage-dong, Nowon-gu, Seoul 602-702, Korea; Phone: 82-02-970-8244; Fax: 02-970-8244; E-mail: eunjoopark.emc@yahoo.com.

(c) Ivyspring International Publisher. This is an open access article distributed under the terms of the Creative Commons Attribution (CC BY-NC) license (https://creativecommons.org/licenses/by-nc/4.0/). See http://ivyspring.com/terms for full terms and conditions.

Received: 2017.11.01; Accepted: 2017.12.17; Published: 2018.03.08

\begin{abstract}
Objective: To evaluate the role of inflammatory markers for distinguishing malignant and benign ovarian masses.

Methods: Preoperative demographic, clinicopathologic, and laboratory variables were reviewed in patients with an ovarian mass that was subsequently diagnosed as either epithelial ovarian cancer (EOC) or a benign ovarian mass on histologic analysis. The differences between variables of the two groups were further evaluated. Logistic regression analysis was applied to evaluate variables to predict the presence of EOC.

Results: According to the analysis of 229 patients with EOC, 120 (52.4\%) patients had serous adenocarcinoma. Of the 229 patients, 110 (48.1\%) patients had stage I or II disease and 119 (52.0\%) had stage III or IV disease. There was a significant difference between EOC and benign ovarian mass in median values of variables such as age, white blood cell (WBC) count, hemoglobin concentration, platelet count, cancer antigen 125 (CA125) levels, neutrophil-to-lymphocyte ratio (NLR), platelet-to-lymphocyte ratio (PLR), and lymphocyte-to-monocyte ratio (LMR) (all $P<0.001$, except for $W B C$ count $[P=0.009])$. In addition, there was significant difference in median values of these continuous variables among early-stage EOC, advanced-stage EOC, and benign ovarian mass $(P<0.001$ for all variables). On multivariate logistic regression analysis, age (odds ratio $[O R]=4.14, P<0.001), C A 125$ levels $(O R=9.87, P<0.001), N L R(O R=1.76, P=0.049)$, PLR (OR $=2.41, P=0.004)$, and $L M R(O R=0.51, P=0.024)$ were found to significantly predict the presence of EOC.

Conclusion: The three LMR, NLR, and PLR markers were found to be predictors for the presence of EOC. Further prospective studies to assess these markers as screening tools for the presence of EOC are required.
\end{abstract}

Key words: Inflammation, Biomarkers, Ovarian neoplasms, Early detection of cancer

\section{Introduction}

Although the incidence of ovarian cancer is low, ovarian cancer remains one of the leading causes of cancer death worldwide among women in both economically developed and developing countries [1].
In 2012, reported deaths due to ovarian cancer included an estimated 65,900 and 86,000 in developed and developing countries, respectively [2]. One of the reasons for the poor prognosis of ovarian cancer is 
that most cases are diagnosed late in the course of disease progression [3]. Reasons for this delayed diagnosis include silent growth of the tumor and the challenges associated with preoperative evaluation of an ovarian mass.

Clinical diagnosis of ovarian cancer is primarily carried out with the help of radiologic findings, clinical symptoms, physical examination, and detection of tumor markers [4-6]. The most important radiologic modality is transvaginal ultrasonography, an important component of the risk of malignancy indices (RMIs) [6, 7]. In addition, magnetic resonance imaging, computed tomography, and positron emission tomography-computed tomography are also useful in the detection of ovarian cancer $[8,9]$.

Women commonly report symptoms prior to the diagnosis of ovarian cancer including bloating, increased abdominal size, pelvic pain, and urinary symptoms that may be more indicative of ovarian cancer rather than benign causes. However, it is difficult to distinguish the symptoms of cancer from those associated with benign masses [10]. The sensitivity and specificity of pelvic examinations for the detection of asymptomatic ovarian cancer are poor and do not support physical examination as a screening method [11].

Cancer antigen 125 (CA125) is one of the most extensively validated tumor markers in ovarian cancers $[5,6,9,12]$, and is a part of the multivariate index assay (OVA1) [13], risk of ovarian malignancy algorithm (ROMA) [14], and RMIs [6, 7]. An increase in the level of CA125 may not be observed in early-stage ovarian cancer and so its role as a screening tool appears to be limited [6]. Recently, the predictive value of other markers such as human epididymis protein 4 has been reported [5]; however, these results are still debatable. Lastly, screening tools for ovarian cancer may include multi-marker panels and bioinformatic analysis [15]. However, the performance of these tests for screening when used alone or in combination has been poor. The U.S. Preventive Services Task Force (USPSTF) recommended against screening for ovarian cancer [16]. Currently, no organization recommends screening in asymptomatic, average-risk women for ovarian cancer [15].

Although there are few sensitive and specific tests for preoperative screening of ovarian cancer, a recent promising approach released by the UK Collaborative Trial of Ovarian Cancer Screening (UKCTOCS) suggests a more favorable outcome for ovarian cancer patients undergoing annual multimodal screening using a risk of ovarian cancer algorithm [17].
Tumor-associated inflammation has long been accepted as a key factor in tumorigenesis and tumor growth. The neutrophil-to-lymphocyte ratio (NLR) and platelet-to-lymphocyte ratio (PLR) are considered predictive factors for survival in ovarian cancer. In addition, preoperative NLR and PLR may help distinguish malignant from benign ovarian masses [18-20]; however, there is lack of consensus regarding their value as a screening tool for ovarian cancer [21]. Recently, the LMR, the ratio of absolute neutrophil count (ANC) to the absolute monocyte count (AMC), has been reported to be a predictive factor for survival in early- and advanced-stage epithelial ovarian cancer (EOC) [22, 23]. However, to the best of our knowledge, there are no published reports on the cut-off LMR value distinguishing malignant from benign ovarian masses. Hence, the aim of present study was to evaluate preoperative LMR as a potential screening tool for EOC.

\section{Methods}

We retrospectively reviewed medical records of patients who had undergone surgical exploration for an ovarian mass by highly trained gynecologic oncologists at university hospitals between July 2003 and September 2016. Patients with either EOC or benign ovarian masses were eligible for inclusion in the study. Patients with the presence of concurrent primary cancers or those having a history of cancer within 5 years of the ovarian mass exploration were excluded from the study. Those patients who had undergone any type of radiation therapy or chemotherapy before surgical exploration were also excluded. Moreover, patients with coexisting autoimmune diseases or with evidence of active infection were excluded.

For consistency, a single, expert pathologist reviewed the histology-based type classification of the ovarian masses. The stage of disease according to the International Federation of Gynecologists and Obstetricians (FIGO) was acquired for analysis. The age of the patients at the time of surgical exploration was obtained from the medical records. Laboratory variables including CA125 levels, white blood cell (WBC) count, hemoglobin $(\mathrm{Hb})$ concentration, platelet count, ANC, absolute lymphocyte count (ALC), and AMC were obtained from the patient medical records. Variables such as NLR, PLR, and LMR were calculated by dividing ANC by ALC, platelet count by ALC, and ALC by AMC, respectively. Data were collected for only those laboratory measurements that were measured prior to surgical resection. If numerous measurements prior to surgery were available, the one that was performed on the date closest to the surgical resection was selected for 
analysis. Quality control criteria and reference ranges adopted at each institution were taken into consideration while collecting the laboratory results. The Mann-Whitney-U test was used to compare the medians between two groups, while comparison of medians between three or more groups of subjects were performed using the Kruskal-Wallis test. A $P$-value of less than 0.05 was regarded as statistically significant.

The initial set of variables in the logistic regression consisted of age, WBC count, $\mathrm{Hb}$ concentration, platelet count, CA125 level, NLR, PLR, and LMR. Variables such as age, WBC count, $\mathrm{Hb}$ concentration, platelet count, and CA125 level were dichotomized based on the predefined cut-off values. However, the optimum cut-off points of NLR, PLR, and LMR for predicting the presence of EOC were determined using receiver operating characteristic (ROC) curve analysis. Subsequently, logistic regression analyses were used to evaluate variables predictive of the presence of EOC. Multivariate analysis was carried out incorporating variables that reached significance in univariate analysis. In addition, all continuous variables were tested using the Pearson's correlation. The SPSS, version 18.0 (SPSS Inc., Chicago, IL, USA), and R-packages were used for data analysis.

\section{Results}

Based on data collected from 229 patients with EOC, the most frequent histology noted was serous adenocarcinoma $(52.4 \%)$, followed by mucinous $(22.3 \%)$, clear cell $(12.7 \%)$, and endometrioid $(10.5 \%)$ types. The most frequent histologic grade in our cohort was grade $3(45.4 \%)$, followed by grade 2 $(31.0 \%)$, and grade $1(23.6 \%)$. In total, $92(40.2 \%)$ patients had stage I, $18(7.9 \%)$ had stage II, 103 (45.9\%) had stage III, and $16(7.0 \%)$ had stage IV disease.

There was a significant difference between EOC and benign ovarian masses in terms of the median values of age, $\mathrm{WBC}$ count, $\mathrm{Hb}$ concentration, platelet count, CA125 level, NLR, PLR, and LMR (all $P<0.001$, except for WBC count $[P=0.009])$ (Table 1). A significant difference was also noted among the three groups, early-stage EOC (stage I or II), advanced-stage (stage III or IV) EOC, and benign ovarian masses, in relation to the aforementioned variables (all $P<0.001$ ). On applying Bonferroni correction, there was a significant difference in medians between advanced-stage EOC and benign ovarian masses in terms of age, WBC count, $\mathrm{Hb}$ concentration, platelet count, CA125 level, NLR, PLR, and LMR. However, a significant difference between early stage EOC and benign ovarian masses was noted in terms of age, Hb concentration, CA125 level,
NLR, PLR, and LMR. Finally, there was a significant difference in medians between early- and advanced-stage EOC in terms of age, WBC count, platelet count, CA125 level, NLR, PLR, and LMR (Table 2).

Table 1. The difference in median values between benign ovarian mass and EOC according to laboratory variables

\begin{tabular}{|c|c|c|c|}
\hline & $\begin{array}{l}\text { Benign ovarian mass, } \\
\text { median (IQR) }(n=261)\end{array}$ & $\begin{array}{l}\text { EOC, } \\
\text { median (IQR) }(n=229)\end{array}$ & $P$ value \\
\hline Age (years) & $35.0(21.0)$ & $54.0(16.0)$ & $<0.001$ \\
\hline WBC (per $\mu \mathrm{L}$ ) & $6220.0(2350.0)$ & $6650.0(2750.0)$ & 0.009 \\
\hline $\begin{array}{l}\text { Hemoglobin } \\
(\mathrm{g} / \mathrm{dL})\end{array}$ & $13.0(1.4)$ & $12.5(1.8)$ & $<0.001$ \\
\hline $\begin{array}{l}\text { Platelets } \\
\left(\times 10^{3} / \mu \mathrm{L}\right)\end{array}$ & $253.0(75.0)$ & $285.0(124.0)$ & $<0.001$ \\
\hline CA125 (unit/mL) & $19.0(21.0)$ & $194.2(541.5)$ & $<0.001$ \\
\hline NLR & $1.9(1.1)$ & $2.8(2.5)$ & $<0.001$ \\
\hline PLR & $136.1(64.2)$ & $190.1(138.5)$ & $<0.001$ \\
\hline LMR & $5.4(2.9)$ & $4.0(3.1)$ & $<0.001$ \\
\hline \multicolumn{4}{|c|}{$\begin{array}{l}P \text {-values for comparisons of medians were obtained using the Mann-Whitney-U } \\
\text { test. } \\
\text { EOC, epithelial ovarian cancer; IQR, interquartile range; WBC, white blood cell; } \\
\text { CA125, cancer antigen } 125 ; \text { NLR, neutrophil-lymphocyte ratio; PLR, } \\
\text { platelet-lymphocyte ratio; LMR, lymphocyte-monocyte ratio }\end{array}$} \\
\hline
\end{tabular}

Table 2. The difference in median values between benign ovarian masses and early-or advanced-stage EOC according to laboratory variables

\begin{tabular}{|c|c|c|c|c|}
\hline & \multirow{2}{*}{$\begin{array}{l}\text { Benign ovarian } \\
\text { mass, median } \\
(\mathrm{IQR})(n=261)\end{array}$} & \multicolumn{2}{|c|}{ EOC, median (IQR) } & \multirow[t]{2}{*}{$P$ value } \\
\hline & & $\begin{array}{l}\text { Stage I-II } \\
(n=110)\end{array}$ & $\begin{array}{l}\text { Stage III-IV } \\
(n=119)\end{array}$ & \\
\hline Age (years) & $35.0(21.0)^{\mathrm{a}}$ & $51.0(14.0)^{\mathrm{b}}$ & $57.0(15.5)^{c}$ & $<0.001$ \\
\hline WBC (per $\mu \mathrm{L})$ & $6220.0(2350.0)^{\mathrm{a}}$ & $6215.0(2600.0)^{\mathrm{a}}$ & $6840.0(3090.0)^{b}$ & $<0.001$ \\
\hline $\begin{array}{l}\text { Hemoglobin } \\
(\mathrm{g} / \mathrm{dL})\end{array}$ & $13.0(1.4)^{\mathrm{a}}$ & $12.6(1.5)^{\mathrm{b}}$ & $12.3(2.0)^{\mathrm{b}}$ & $<0.001$ \\
\hline $\begin{array}{l}\text { Platelets } \\
\left(\times 10^{3} / \mu \mathrm{L}\right)\end{array}$ & $253.0(75.0)^{\mathrm{a}}$ & $271.5(95.0)^{a}$ & $328.0(152.5)^{b}$ & $<0.001$ \\
\hline $\begin{array}{l}\text { CA125 } \\
\text { (unit/mL) }\end{array}$ & $19.0(21.0)^{a}$ & $52.7(227.9)^{\mathrm{b}}$ & $471.6(751.2)^{c}$ & $<0.001$ \\
\hline NLR & $1.9(1.1)^{\mathrm{a}}$ & $2.4(1.7)^{\mathrm{b}}$ & $3.5(3.0)^{c}$ & $<0.001$ \\
\hline PLR & $136.1(64.2)^{a}$ & $163.4(93.1)^{b}$ & $234.3(195.0)^{c}$ & $<0.001$ \\
\hline LMR & $5.4(2.9)^{\mathrm{a}}$ & $4.8(3.1)^{\mathrm{b}}$ & $3.2(2.6)^{c}$ & $<0.001$ \\
\hline
\end{tabular}

Medians with the same letter (superscript) are not significantly different. $P$-values for comparisons of medians were obtained using the Kruskal-Wallis test. A post-hoc test (Bonferroni) was applied with pairwise comparison between medians. EOC, epithelial ovarian cancer; IQR, interquartile range; WBC, white blood cell; CA125, cancer antigen 125; NLR, neutrophil-lymphocyte ratio; PLR, platelet-lymphocyte ratio; LMR, lymphocyte-monocyte ratio

Using data from all eligible patients, the best cut-off points based on ROC curve analyses for NLR, PLR, and LMR were found to be 2.64 (AUC $=0.709$; sensitivity 0.568 , specificity $0.774 ; P<0.001), 191.71$ (AUC $=0.718 ; \quad$ sensitivity 0.498 , specificity 0.843 ; $P<0.001$ ) and 3.52 (AUC $=0.683$; sensitivity 0.437, specificity $0.862 ; P<0.001$ ), respectively (Figure 1 ). Univariate logistic regression analyses identified the following significant variables for EOC: age, WBC count, $\mathrm{Hb}$ concentration, CA125 level, NLR, PLR, and LMR (all $P<0.001$, except for WBC count $[P=0.015]$ ). On multivariate logistic regression analyses, age 
(odds ratio $[\mathrm{OR}]=4.14,95 \%$ confidence interval $[C I]=2.00-8.90, P<0.001), C A 125$ levels $(\mathrm{OR}=9.87,95 \%$ $\mathrm{CI}=6.27-15.84, \quad P<0.001), \quad$ NLR $\quad(\mathrm{OR}=1.76, \quad 95 \%$ $\mathrm{CI}=1.00-3.09, \quad P=0.049), \quad$ PLR $\quad(\mathrm{OR}=2.41, \quad 95 \%$ $\mathrm{CI}=1.34-4.38, \quad P=0.004)$ and LMR (OR=0.51, 95\% $\mathrm{CI}=0.28-0.91, P=0.024)$ were found to predict the presence of EOC (Table 3 and Figure 2).

The correlation between variables is shown in Figure 3 . There was a moderate negative correlation

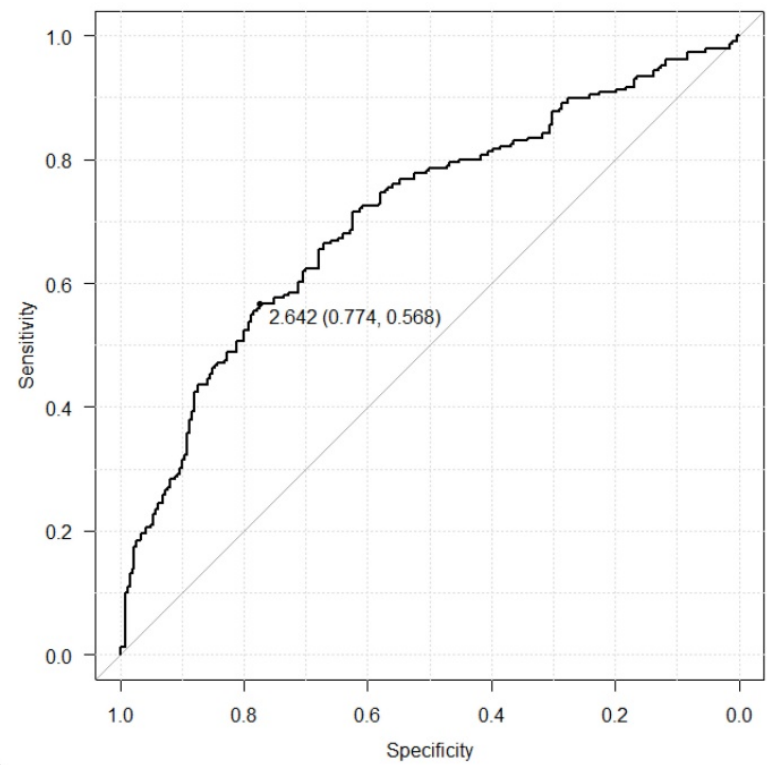

(A)

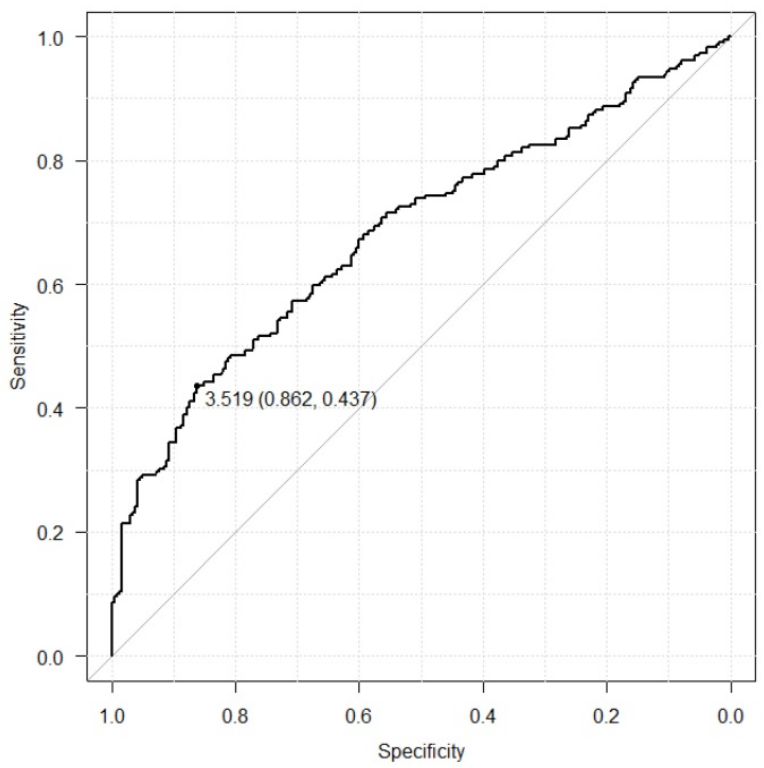

between LMR and NLR $(r=-0.43, P<0.001)$ or PLR $(r=-0.31, P<0.001)$, weak negative correlation between LMR and CA125 level $(r=-0.26, P<0.001)$, very weak negative correlation between LMR and age $(r=-0.16$. $P<0.001)$ or WBC count $(r=-0.19, P<0.001)$, and very weak positive correlation between $\mathrm{LMR}$ and $\mathrm{Hb}$ concentration $(r=0.19, P<0.001)$.

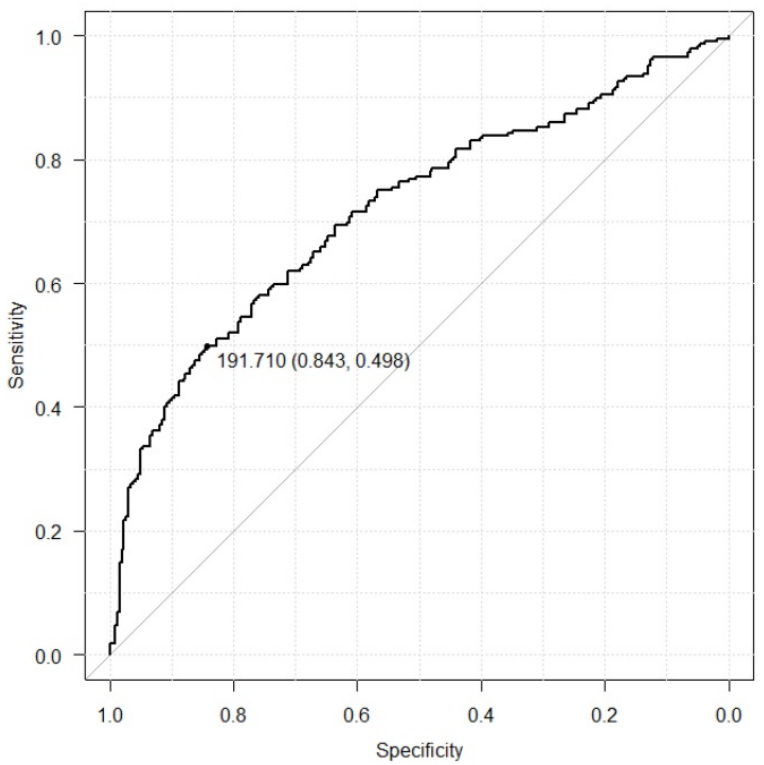

(B)

Figure 1. Receiver operating characteristic (ROC) curves for (A) neutrophil-lymphocyte ratio (NLR), (B) platelet-lymphocyte ratio (PLR), and (C) lymphocyte-monocyte ratio (LMR). The numbers before the parentheses depict cut-off values, and the numbers in the parentheses show specificity and sensitivity in order. 
Table 3. Univariate and multivariate analyses for the evaluation of variables that predict the presence of EOC

\begin{tabular}{|c|c|c|c|c|}
\hline & Univariate & & Multivariate & \\
\hline & OR $(95 \% \mathrm{CI})$ & $P$ value & OR $(95 \% \mathrm{CI})$ & $P$ value \\
\hline Age (years) (<65 vs. $\geq 65)$ & $4.39(2.47,8.18)$ & $<0.001$ & $4.14(2.00,8.90)$ & $<0.001$ \\
\hline WBC (per $\mu \mathrm{L})(\leq 11000$ vs. $>11000)$ & $2.86(1.27,7.06)$ & 0.015 & & \\
\hline Hemoglobin (g/dL) ( $\leq 12.0$ vs. >12.0) & $0.42(0.28,0.64)$ & $<0.001$ & & \\
\hline Platelets $\left(\times 10^{3}\right.$ per $\left.\mu \mathrm{L}\right)(\leq 400$ vs. $>400)$ & $7.93(3.52,21.26)$ & $<0.001$ & & \\
\hline CA125 (unit/mL) ( $\leq 35$ vs. $>35)$ & $12.58(8.26,19.49)$ & $<0.001$ & $9.87(6.27,15.84)$ & $<0.001$ \\
\hline $\operatorname{NLR}(\leq 2.64$ vs. $>2.64)$ & $4.50(3.06,6.68)$ & $<0.001$ & $1.76(1.00,3.09)$ & 0.049 \\
\hline PLR ( $\leq 191.71$ vs. $>191.71)$ & $5.68(3.70,8.88)$ & $<0.001$ & $2.41(1.34,4.38)$ & 0.004 \\
\hline LMR ( $\leq 3.52$ vs. $>3.52)$ & $0.21(0.14,0.33)$ & $<0.001$ & $0.51(0.28,0.91)$ & 0.024 \\
\hline
\end{tabular}

Results of multiple logistic regression with variables show a $P$-value less than 0.05 in univariate regression.

EOC, epithelial ovarian cancer; OR, odds ratio; CI, confidence interval; WBC, white blood cell; CA125, cancer antigen 125; NLR, neutrophil-lymphocyte ratio; PLR, platelet-lymphocyte ratio; LMR, lymphocyte-monocyte ratio

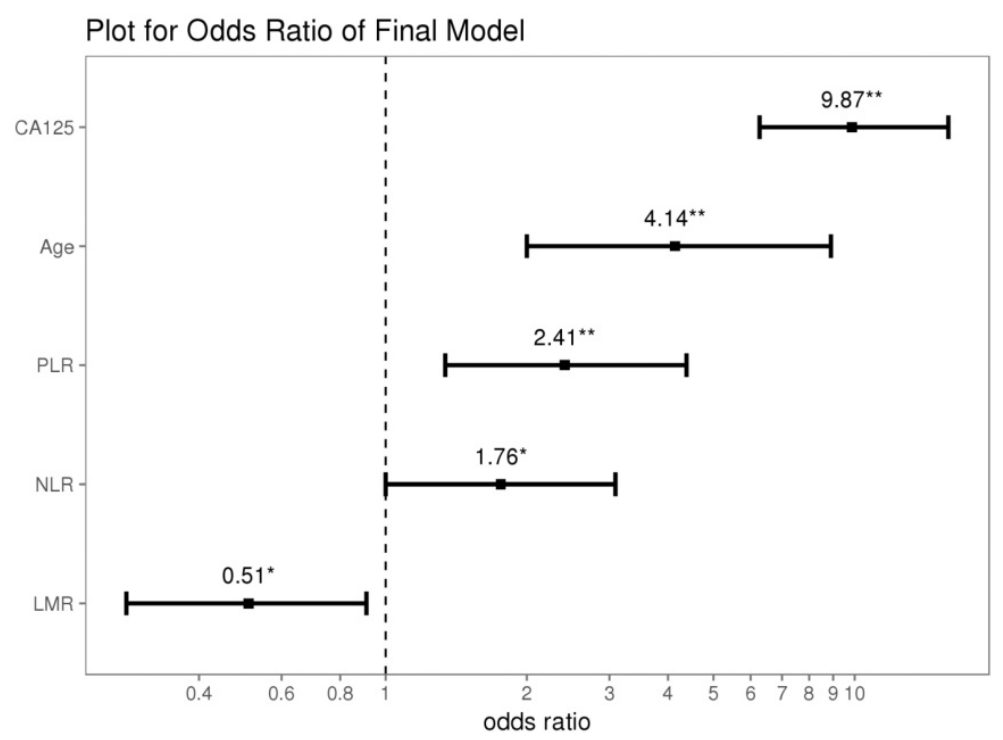

Figure 2. Results of the stepwise backward regression. * $P<0.05$; ** $P<0.01$; CA125, cancer antigen 125; NLR, neutrophil-lymphocyte ratio; PLR, platelet-lymphocyte ratio; LMR, lymphocyte-monocyte ratio

\begin{tabular}{|l|l|l|l|l|l|l|l|l|}
\hline Variable & Age & WBC & Hb & Platelet & CA125 & NLR & PLR & LMR \\
\hline Age & 1 & & & & & & & \\
\hline WBC & -0.01 & 1 & & & & & & \\
\hline $\mathrm{Hb}$ & $-0.11^{*}$ & 0.07 & 1 & & & & & \\
\hline Platelets & 0.08 & $0.30^{* * *}$ & $-0.15^{* *}$ & 1 & & & & \\
\hline CA125 & $0.30^{* * *}$ & $0.19^{* * *}$ & $-0.23^{* * *}$ & $0.27^{* * *}$ & 1 & & & \\
\hline NLR & $0.15^{* *}$ & $0.51^{* * *}$ & $-0.13^{* *}$ & $0.17^{* * *}$ & $0.31^{* * *}$ & 1 & & \\
\hline PLR & $0.16^{* * *}$ & 0.01 & $-0.22^{* * *}$ & $0.54^{* * *}$ & $0.33^{* * *}$ & $0.54^{* * *}$ & 1 & \\
\hline LMR & $-0.16^{* * *}$ & $-0.19^{* * *}$ & $0.19^{* * *}$ & -0.09 & $-0.26^{* * *}$ & $-0.43^{* * *}$ & $-0.31^{* * *}$ & 1 \\
\hline
\end{tabular}

$* * * P<0.001, * * P<0.01, * P<0.05$

WBC, white blood cell; Hb, hemoglobin; CA125, cancer antigen 125; NLR, neutrophil-lymphocyte ratio; PLR, platelet-lymphocyte ratio; LMR, lymphocyte-monocyte ratio

Figure 3. The ' $r$ ' values by Pearson's product-moment correlation

\section{Discussion}

Although previous studies have reported various potential tools to predict the presence of ovarian cancer, the results are debatable, with no evidence in support of tools that can distinguish ovarian cancer from benign ovarian masses $[15,16]$. In the present study, we specifically evaluated inflammatory markers and found that LMR, along with NLR and PLR, could predict the presence of EOC.

Monocytes from patients with advanced cancer are considered to display immunosuppressive properties [24]. In addition, the monocyte count of the peripheral blood has an association with the density of the tumor-associated macrophages, which creates a favorable microenvironment for the development of 
cancer [25]. An elevated monocyte count in peripheral blood has been associated with detrimental outcomes for cancer patients. LMR is calculated as the ratio of ALC and AMC, and it has been proposed as a prognostic or predictive factor associated with survival in various malignancies including ovarian cancers [22, 23, 26, 27]. In EOC with stage I to IV disease, a low LMR is an independent predictor for overall survival (OS) [22]. In addition, a low LMR is a significant prognostic factor associated with adverse progression-free survival (PFS) and OS in advanced EOC (stage III and IV) [23]; the cut-off values of the previous studies range from 2.07 to 3.45 . Furthermore, preoperative LMR, with a cut-off value of 3.75 , is reported to be an independent predictor associated with suboptimal cytoreduction in stage III and IV EOC [27]. However, there are no studies describing the LMR cut-off value as a screening tool for EOC. In our study, the LMR with a cut-off value of 3.52 predicted the presence of EOC, along with NLR and PLR.

The NLR has been recognized as a potent prognosticator for PFS $[28,29]$ and OS $[19,29,30]$ in ovarian cancer. In addition, NLR has been suggested to predict the presence of ovarian cancer. In previous studies, the cut-off values based on ROC curve analysis have ranged from 3.45 to $3.47[18,20]$. In our study the cut-off value by ROC curve analysis was 2.64; we also demonstrated that NLR predicts the presence of EOC. However, the value of NLR that can predict the presence of EOC needs further evaluation, as a significant conclusion could not be reached at the end of the present study.

The peripheral blood platelet count is reportedly increased in $31 \%$ to $56 \%$ of adnexal tumors [31]. Although the underlying mechanism of increased platelet counts is not well understood, increased hepatic thrombopoietin has been suggested as one of the contributors [32]. Platelets are actively involved in the growth of ovarian cancer cells [33], and the change in the platelet count itself may control tumor growth in ovarian cancers. Decreasing platelet count using anti-platelet antibodies prevents the growth of ovarian cancer [32], and increasing the platelet count via platelet transfusion increases the size of the tumor [34]. In addition, elevated platelet count in the peripheral blood is associated with aggressive behavior and advanced stages of ovarian cancer [32, 35]. Furthermore, the peripheral blood platelet count is suggested to predict the presence of ovarian cancer $[31,36,37]$. In our study, however, platelet count did not predict the presence of EOC. A possible explanation for this anomaly could be the analysis of the platelet count with PLR, a more potent systemic inflammatory response marker derived from the platelet count and ALC. The PLR has been associated with the prognosis of ovarian cancer [38, 39]. In addition, use of the PLR as a screening tool for the diagnosis of ovarian cancer has been reported; however, the number of patients enrolled in those studies was too small for clinical translation $[18,20]$, and one of those studies included only advanced stage disease (stage IIIc or IV) [18]. On ROC curve analysis, the cut-off values in the previous studies ranged from 161.13 to $572.9[18,20]$. In our study, the PLR with a cut-off value of 191.71 , by ROC curve analysis, predicted the presence of EOC.

In this study, age also predicted the presence of EOC. Several researchers have suggested age to be an independent demographic variable to distinguish malignant from benign ovarian masses [20, 31, 40, 41], and our finding was compatible with these reports. In addition to age, menopausal status has been adopted to form the ROMA that classifies an adnexal mass into high- or low-risk EOC groups [14]. In our study, however, we could not analyze the role of menopausal status due to missing values.

CA125 is the one of the most extensively validated biomarkers in ovarian cancers $[5,6,9,12,31$, 41]. Approximately $80 \%$ of women with EOC have CA125 levels exceeding the cut-off value of $35 \mathrm{kU} / \mathrm{L}$, with elevations of $50-60 \%, 80-90 \%$, and $>90 \%$ in clinical stage I, II, and III-IV disease, respectively [6]. The frequency of elevated concentrations is the highest in patients with serous EOC followed by endometrioid, clear cell, and mucinous types [6]. In the clinic, CA125 has been used as a laboratory tool for monitoring response to first-line chemotherapy [6]. In addition, CA125 is a part of OVA1 [13], ROMA [14], and RMI [6, 7]. Furthermore, preoperative CA125 levels can be used to predict the presence of ovarian cancer $[6,7,13,14,20,31,40-45]$, and the result of our study is compatible with these studies. However, CA125 is not currently recommended as a screening tool for ovarian cancer in asymptomatic patients because of its low sensitivity and limited specificity, and due to the fact that increased CA125 levels may not observed in early-stage ovarian or mucinous type cancer [6]. In the present study, analysis of CA125 levels in serous and non-serous types revealed no significant differences in CA125 level according to histologic type in advanced-stage EOC (median, 492.6 unit/mL with an interquartile range [IQR] of 725.2 unit $/ \mathrm{mL}$ in the serous type and median, 444.6 unit/mL with IQR of $795.1 \mathrm{unit} / \mathrm{mL}$ in the non-serous type; $P=0.768)$. However, there was a significant difference in CA125 levels in early-stage EOC according to histologic type (median, 240.6 unit/mL with IQR of 403.4 unit/mL in the serous type and median, 32.6 unit $/ \mathrm{mL}$ with IQR of 91.9 unit $/ \mathrm{mL}$ in the 
non-serous type; $P<0.001)$. When considering the pre-defined CA125 cut-off value of 35 unit/mL in ovarian cancer, our results may limit the clinical application to patients with non-serous histology and early-stage EOC. In addition, drawbacks of the CA125 biomarker include its elevation in various benign gynecologic diseases such as uterine myoma, adenomyosis, endometriosis, salpingitis, and ovarian cysts and in several non-gynecological diseases such as pelvic inflammatory disease, liver cirrhosis, acute hepatitis, and pancreatitis, and in peritoneal, pleural, and musculoskeletal diseases. Additionally, elevated concentrations can also occur in other malignancies such as hepatocellular carcinoma and advanced adenocarcinomas of the pancreas, biliary tract, lungs, endometrium, stomach, cervix, breasts, and colorectal areas $[6,46]$.

To the best of our knowledge, this is the first study to identify LMR as a predictor of the presence of both early- and advanced-stage EOC in patients with an ovarian mass. Specifically, in the present study, the value of $L M R$ as an independent predictive factor associated with the presence of EOC was demonstrated by analyzing it together with other inflammatory markers, such as NLR and PLR, using multivariate analysis. However, the main limitation of the study is the fact that it is a retrospective cohort study. In addition, although we excluded patients with the coexistence of autoimmune diseases or evidence of active infection, the diverse systemic diseases or various inflammatory conditions may have affected the LMR values [47].

In conclusion, LMR along with NLR, PLR, age, and CA125 levels could predict the presence of EOC in our study. The results of our study indicate that in addition to the previously validated biomarkers, there exists a potential role of LMR as a predictor of the presence of EOC. However, it is too early to apply LMR as a screening tool in the general population at present and additional large-scale prospective investigations to determine the utility of such predictive biomarkers are clearly warranted.

\section{Competing Interests}

The authors have declared that no competing interest exists.

\section{References}

(1) Siegel R, Naishadham D, Jemal A. Cancer statistics, 2013. CA: a cancer journal for clinicians. 2013;63:11-30.

(2) Torre LA, Bray F, Siegel RL, Ferlay J, Lortet-Tieulent J, Jemal A. Global cancer statistics, 2012. CA: a cancer journal for clinicians. 2015;65:87-108.

(3) Vergote I, Trope CG, Amant F, Kristensen GB, Ehlen T, Johnson N, et al. Neoadjuvant chemotherapy or primary surgery in stage IIIC or IV ovarian cancer. The New England journal of medicine. 2010;363:943-53.

(4) ACOG Practice Bulletin. Management of adnexal masses. Obstet Gynecol. 2007;110:201-14

(5) Nossov V, Amneus M, Su F, Lang J, Janco JM, Reddy ST, et al. The early detection of ovarian cancer: from traditional methods to proteomics. Can we really do better than serum CA-125? American journal of obstetrics and gynecology. 2008;199:215-23.

(6) Soletormos G, Duffy MJ, Othman Abu Hassan S, Verheijen RH, Tholander B, Bast RC, Jr., et al. Clinical Use of Cancer Biomarkers in Epithelial Ovarian Cancer: Updated Guidelines From the European Group on Tumor Markers. Int J Gynecol Cancer. 2016;26:43-51.

(7) Manegold-Brauer G, Bellin AK, Tercanli S, Lapaire O, Heinzelmann-Schwarz $\mathrm{V}$. The special role of ultrasound for screening, staging and surveillance of malignant ovarian tumors: distinction from other methods of diagnostic imaging. Arch Gynecol Obstet. 2014;289:491-8.

(8) Medeiros LR, Freitas LB, Rosa DD, Silva FR, Silva LS, Birtencourt LT, et al. Accuracy of magnetic resonance imaging in ovarian tumor: a systematic quantitative review. American journal of obstetrics and gynecology. 2011;204:67.e1-10.

(9) Nam EJ, Yun MJ, Oh YT, Kim JW, Kim JH, Kim S, et al. Diagnosis and staging of primary ovarian cancer: correlation between PET/CT, Doppler US, and CT or MRI. Gynecol Oncol. 2010;116:389-94.

(10) Goff BA, Mandel LS, Melancon CH, Muntz HG. Frequency of symptoms of ovarian cancer in women presenting to primary care clinics. JAMA : the journal of the American Medical Association. 2004;291:2705-12.

(11) Bloomfield HE, Olson A, Greer N, Cantor A, MacDonald R, Rutks I, et al. Screening pelvic examinations in asymptomatic, average-risk adult women: an evidence report for a clinical practice guideline from the American College of Physicians. Annals of internal medicine. 2014;161:46-53.

(12) Bozkurt M, Yumru AE, Aral I. Evaluation of the importance of the serum levels of CA-125, CA15-3, CA-19-9, carcinoembryonic antigen and alpha fetoprotein for distinguishing benign and malignant adnexal masses and contribution of different test combinations to diagnostic accuracy. European journal of gynaecological oncology. 2013;34:540-4.

(13) Bristow RE, Smith A, Zhang Z, Chan DW, Crutcher G, Fung ET, et al. Ovarian malignancy risk stratification of the adnexal mass using a multivariate index assay. Gynecol Oncol. 2013;128:252-9.

(14) Moore RG, Miller MC, Disilvestro P, Landrum LM, Gajewski W, Ball JJ, et al. Evaluation of the diagnostic accuracy of the risk of ovarian malignancy algorithm in women with a pelvic mass. Obstet Gynecol. 2011;118:280-8.

(15) Smith RA, Andrews KS, Brooks D, Fedewa SA, Manassaram-Baptiste D, Saslow D, et al. Cancer screening in the United States, 2017: A review of current American Cancer Society guidelines and current issues in cancer screening. CA: a cancer journal for clinicians. 2017;67:100-21.

(16) Summaries for patients. Screening for ovarian cancer: U.S. Preventive Services Task Force reaffirmation recommendation statement. Annals of internal medicine. 2012;157:I-56.

(17) Jacobs IJ, Menon U, Ryan A, Gentry-Maharaj A, Burnell M, Kalsi JK, et al. Ovarian cancer screening and mortality in the UK Collaborative Trial of Ovarian Cancer Screening (UKCTOCS): a randomised controlled trial. Lancet. 2016;387:945-56.

(18) Yildirim M, Demir Cendek B, Filiz Avsar A. Differentiation between benign and malignant ovarian masses in the preoperative period using neutrophil-to-lymphocyte and platelet-to-lymphocyte ratios. Molecular and clinical oncology. 2015;3:317-21.

(19) Cho H, Hur HW, Kim SW, Kim SH, Kim JH, Kim YT, et al. Pre-treatment neutrophil to lymphocyte ratio is elevated in epithelial ovarian cancer and predicts survival after treatment. Cancer immunology, immunotherapy : CII. 2009;58:15-23.

(20) Bakacak M, Serin S, Ercan O, Kostu B, Bostanci MS, Bakacak Z, et al. Utility of preoperative neutrophil-to-lymphocyte and platelet-to-lymphocyte ratios to distinguish malignant from benign ovarian masses. Journal of the Turkish German Gynecological Association. 2016;17:21-5.

(21) Prodromidou A, Andreakos P, Kazakos C, Vlachos DE, Perrea D, Pergialiotis V. The diagnostic efficacy of platelet-to-lymphocyte ratio and neutrophil-to-lymphocyte ratio in ovarian cancer. Inflammation research : official journal of the European Histamine Research Society [et al]. 2017.

(22) Eo WK, Chang HJ, Kwon SH, Koh SB, Kim YO, Ji YI, et al. The Lymphocyte-Monocyte Ratio Predicts Patient Survival and Aggressiveness of Ovarian Cancer. Journal of Cancer. 2016;7:289-96.

(23) Zhu JY, Liu CC, Wang L, Zhong M, Tang HL, Wang H. Peripheral blood lymphocyte-to-monocyte ratio as a prognostic factor in advanced epithelial ovarian cancer: a multicenter retrospective study. Journal of Cancer. 2017;8:737-43.

(24) Bergenfelz C, Larsson AM, von Stedingk K, Gruvberger-Saal S, Aaltonen K, Jansson S, et al. Systemic Monocytic-MDSCs Are Generated from Monocytes and Correlate with Disease Progression in Breast Cancer Patients. PloS one. 2015;10:e0127028.

(25) Shibutani M, Maeda K, Nagahara H, Fukuoka T, Nakao S, Matsutani S, et al. The peripheral monocyte count is associated with the density of tumor-associated macrophages in the tumor microenvironment of colorectal cancer: a retrospective study. BMC cancer. 2017;17:404.

(26) Porrata LF, Ristow K, Colgan JP, Habermann TM, Witzig TE, Inwards DJ, et al. Peripheral blood lymphocyte/monocyte ratio at diagnosis and survival in classical Hodgkin's lymphoma. Haematologica. 2012;97:262-9.

(27) Eo W, Kim HB, Lee YJ, Suh DS, Kim KH, Kim H. Preoperative Lymphocyte-Monocyte Ratio Is a Predictor of Suboptimal Cytoreduction in Stage III-IV Epithelial Ovarian Cancer. Journal of Cancer. 2016;7:1772-9. 
(28) Kim HS, Choi HY, Lee M, Suh DH, Kim K, No JH, et al. Systemic Inflammatory Response Markers and CA-125 Levels in Ovarian Clear Cell Carcinoma: A Two Center Cohort Study. Cancer Res Treat. 2015.

(29) Wang Y, Liu P, Xu Y, Zhang W, Tong L, Guo Z, et al. Preoperative neutrophil-to-lymphocyte ratio predicts response to first-line platinum-based chemotherapy and prognosis in serous ovarian cancer. Cancer Chemother Pharmacol. 2015;75:255-62.

(30) Williams KA, Labidi-Galy SI, Terry KL, Vitonis AF, Welch WR, Goodman A, et al. Prognostic significance and predictors of the neutrophil-to-lymphocyte ratio in ovarian cancer. Gynecol Oncol. 2014;132:542-50.

(31) Watrowski R, Heinze G, Jager C, Forster J, Zeillinger R. Usefulness of the preoperative platelet count in the diagnosis of adnexal tumors. Tumour biology : the journal of the International Society for Oncodevelopmental Biology and Medicine. 2016;37:12079-87.

(32) Stone RL, Nick AM, McNeish IA, Balkwill F, Han HD, Bottsford-Miller J, et al. Paraneoplastic thrombocytosis in ovarian cancer. The New England journal of medicine. 2012;366:610-8.

(33) Cho MS, Bottsford-Miller J, Vasquez HG, Stone R, Zand B, Kroll MH, et al. Platelets increase the proliferation of ovarian cancer cells. Blood. 2012;120:4869-72.

(34) Bottsford-Miller J, Choi HJ, Dalton HJ, Stone RL, Cho MS, Haemmerle M, et al. Differential platelet levels affect response to taxane-based therapy in ovarian cancer. Clinical cancer research : an official journal of the American Association for Cancer Research. 2015;21:602-10.

(35) Li AJ, Madden AC, Cass I, Leuchter RS, Lagasse LD, Karlan BY. The prognostic significance of thrombocytosis in epithelial ovarian carcinoma. Gynecol Oncol. 2004;92:211-4.

(36) Chalas E, Welshinger M, Engellener W, Chumas J, Barbieri R, Mann WJ. The clinical significance of thrombocytosis in women presenting with a pelvic mass. American journal of obstetrics and gynecology. 1992;166:974-7.

(37) Atacag T. Diagnostic value of thrombocytosis and high CA 125 level in women with adnexal masses. European journal of gynaecological oncology. 2012;33:517-20.

(38) Asher V, Lee J, Innamaa A, Bali A. Preoperative platelet lymphocyte ratio as an independent prognostic marker in ovarian cancer. Clinical \& translational oncology : official publication of the Federation of Spanish Oncology Societies and of the National Cancer Institute of Mexico. 2011;13:499-503.

(39) Raungkaewmanee S, Tangiitgamol S, Manusirivithaya S, Srijaipracharoen S, Thavaramara T. Platelet to lymphocyte ratio as a prognostic factor for epithelial ovarian cancer. J Gynecol Oncol. 2012;23:265-73.

(40) Watrowski R, Zeillinger R. Simple laboratory score improves the preoperative diagnosis of adnexal mass. Tumour biology : the journal of the International Society for Oncodevelopmental Biology and Medicine. 2016;37:4343-9.

(41) Wilailak S, Chan KK, Chen CA, Nam JH, Ochiai K, Aw TC, et al. Distinguishing benign from malignant pelvic mass utilizing an algorithm with HE4, menopausal status, and ultrasound findings. J Gynecol Oncol. 2015;26:46-53.

(42) Topcu HO, Guzel AI, Ozer I, Kokanali MK, Gokturk U, Muftuoglu KH, et al. Comparison of neutrophil/lymphocyte and platelet/ lymphocyte ratios for predicting malignant potential of suspicious ovarian masses in gynecology practice. Asian Pacific journal of cancer prevention : APJCP. 2014;15:6239-41.

(43) Miyagi E, Maruyama Y, Mogami T, Numazaki R, Ikeda A, Yamamoto H, et al. Comparison of plasma amino acid profile-based index and CA125 in the diagnosis of epithelial ovarian cancers and borderline malignant tumors. International journal of clinical oncology. 2017:22:118-25.

(44) Cymbaluk-Ploska A, Chudecka-Glaz A, Surowiec A, Pius-Sadowska E, Machalinski B, Menkiszak J. MMP3 in Comparison to CA 125, HE4 and the ROMA Algorithm in Differentiation of Ovarian Tumors. Asian Pacific journal of cancer prevention : APJCP. 2016;17:2597-603.

(45) Cho HY, Park SH, Park YH, Kim HB, Kang JB, Hong SH, et al. Comparison of HE4, CA125, and Risk of Ovarian Malignancy Algorithm in the Prediction of Ovarian Cancer in Korean Women. Journal of Korean medical science. 2015;30:1777-83.

(46) Moss EL, Hollingworth J, Reynolds TM. The role of CA125 in clinical practice. Journal of clinical pathology. 2005;58:308-12.

(47) Madjid M, Fatemi O. Components of the complete blood count as risk predictors for coronary heart disease: in-depth review and update. Texas Heart Institute journal / from the Texas Heart Institute of St Luke's Episcopal Hospital, Texas Children's Hospital. 2013;40:17-29. 